\title{
SOME REMARKS ON $l-l$ SUMMABILITY
}

BY H. I. BROWN AND D. R. KERR

Communicated by Everett Pitcher, December 5, 1967

Introduction. If $x$ is a complex number sequence and $A=\left(a_{n k}\right)$ is an infinite matrix of complex numbers, then $A$ determines a transformation of $x$ into the sequence $A x$, where $(A x)_{n}=\sum_{k} a_{n k} x_{k}$. Let $l$ represent the set of complex sequences with finite norm $\|x\|=\sum\left|x_{k}\right|$. If $A x \in l$ whenever $x \in l$, then $A$ is called an $l-l$ method of summation. Let $l_{A}$ denote the summability field of $A$, that is, the set of all sequences $x$ such that $A x \in l$. In [3] an attempt is made to characterize those $l-l$ methods $A$ for which $l_{A}=l$. In what follows we give counterexamples to Theorems 7 and 9 of [3], announce several positive results related to these theorems, and generalize Theorem 5 of [3] for the class of factorable $l-l$ methods.

1. Terminology and notation. Knopp and Lorentz [4] show that the matrix $A$ is an $l-l$ method if and only if $\|A\|<\infty$, where $\|A\|$ $=\sup _{k} \sum_{n}\left|a_{n k}\right| .\|A\|$ is the norm of $A$ as an operator from $l$ to $l$. It is known that $l_{A}$ inherits a locally convex topology making it an FK space. Moreover, each $f \in l_{A}^{\prime}$, the dual space of $l_{A}$, has the representation

$$
f(x)=\sum_{n} t_{n} \sum_{k} a_{n k} x_{k}+\sum_{k} \beta_{k} x_{k}
$$

for some bounded sequences $t$ and $\beta$. An $l-l$ method $A$ is reversible if the equation $y=A x$ has a unique solution $x$ in $l_{A}$ for each $y$ in $l$. An $l-l$ method $A$ is called perfect if $l$ is dense in $l_{A}$ (equivalently, if the set $\Delta=\left\{e^{k}: k=1,2, \cdots\right\}$, where $e^{k}$ is the sequence having a one in the $k$ th coordinate and zeros elsewhere, is fundamental in $l_{A}$ ). Let $m_{r}$ denote the set of all sequences $x$ with finite norm $\|x\|=\sup _{m}\left|\sum_{k=1}^{m} x_{k}\right|$. In [3], an $l-l$ method $A$ is called 0 -perfect if every sequence $x$ in $m_{r} \cap l_{A}$ is a limit point in $l_{A}$ of the set $l$. Concerning these concepts, Jürimäe makes the following two statements. (In Statement B he omits the assumption of reversibility.)

Statement A [3, Theorem 7]. If $A$ is an 0 -perfect $l-l$ method with $l_{A} \subseteq m_{r}$, then $l_{A}=l$.

Statement B [3, Theorem 9]. A reversible 0-perfect $l-l$ method $B$ sums a sequence $x \notin l$ if and only if

$$
\sup _{m} \sup _{k}\left|\sum_{n=1}^{m} b_{n k}^{\prime}\right|=\infty \text {, }
$$


where $B^{\prime}=\left(b_{n k}^{\prime}\right)$ is the two-sided reciprocal for $B$. (Given a matrix $B C$ is a two-sided reciprocal for $B$ if $B C=C B=I$, where $I$ is the identity matrix. Right and left reciprocals are defined analogously.)

2. Examples. Let $A=\left(a_{n k}\right)$ be defined by the set of equations

$$
\begin{array}{ll}
a_{1 k}=1 & (k=1,2, \cdots), \\
a_{n k}=0 & \text { otherwise. }
\end{array}
$$

$A$ is an 0-perfect $l-l$ method (indeed, $A$ is perfect; see [2, p. 361]). Moreover, $l_{A}=c_{r}$, where $c_{r}$ is the set of all sequences $x$ for which the series $\sum_{k} x_{k}$ converges. Hence, for 0 -perfect $l-l$ methods, the condition $l_{A} \subseteq m_{r}$ is clearly not sufficient for $l_{A}=l$ as Statement A asserts.

Next let $B=\left(b_{n k}\right)$ be given by the set of equations

$$
\begin{aligned}
& b_{1 k}=1 \quad(k=1,2, \cdots), \\
& b_{n n}=b_{n, n+1}=1 \quad(n=2,3, \cdots), \\
& b_{n k}=0 \quad \text { otherwise. }
\end{aligned}
$$

$B$ is one-to-one on $l_{A}$ because $\left\{\alpha(-1)^{k}\right\} \in l_{A} \subset c_{r}$ if and only if $\alpha=0$. Furthermore, given $y \in l$ choose $x_{k}=\sum_{i=k}^{\infty}(-1)^{i-k} y_{i}$ for $k=2,3, \cdots$, and $x_{1}=y_{1}-\sum_{k=2}^{\infty} \sum_{i=k}^{\infty}(-1)^{i-k} y_{i}$. Then $B x=y$ so that $B$ is onto $l$. Since the zero sequence is the only bounded left annihilator of $B, B$ is perfect [2, Theorem 2]. Its two-sided reciprocal $B^{\prime}=\left(b_{n \mathrm{k}}^{\prime}\right)$ is given by the set of equations

$$
\begin{aligned}
b_{11}^{\prime} & =1, \\
b_{1,2 k}^{\prime} & =-1 \quad(k=1,2, \cdots), \\
b_{1,2 k+1}^{\prime} & =0 \quad(k=1,2, \cdots), \\
b_{n, n+k-1}^{\prime} & =(-1)^{k-1} \quad(k \geqq 1, n \geqq 2), \\
b_{n k}^{\prime} & =0 \quad \text { otherwise. }
\end{aligned}
$$

Clearly, $B^{\prime}$ does not satisfy Equation (1), yet $l_{B}$ properly contains $l$ since $\left\{(-1)^{k} / k\right\} \in l_{B}$; hence, for a reversible 0 -perfect $l-l$ method, Equation (1) is not necessary for $l_{A}=l$ as Statement B asserts.

3. Comments and results concerning $l_{A}=l$. The hypotheses of each of Statements A and B do imply that $l_{A} \subseteq c_{r .}$. This is not surprising since these hypotheses seem to be more natural for $c_{r}-c_{r}$ methods than for $l-l$ methods.

Using a result of Banach [1, p. 49], one can easily show that every reversible $l-l$ method has a unique two-sided reciprocal which is its inverse as an operator from $l_{A}$ to $l$. This result leads immediately to the following theorem. 
TheOREM 1. Let $A$ be a reversible $l-l$ method. Then $l_{A}=l$ if and only if its two-sided reciprocal is an $l-l$ method.

Indeed, since $A^{\prime}(l)=l_{A}, l_{A}=l$ if and only if $A^{\prime}$ is an $l-l$ method.

Using the techniques of FK spaces (see, for example, [5]) one gets the following results concerning left reciprocals.

LEMma 1. If the $l-l$ method $A$ has a left $l-l$ reciprocal $B$, then $l$ is closed in $l_{A}$.

Proof. For $x \in l,\|x\|=\|(B A) x\| \leqq\|B\| \cdot\|A x\|$, where $\|B\|<\infty$ since $B$ is $l-l$. Therefore, the $l_{A}$ topology on $l$ is stronger than the usual topology on $l$.

From this lemma we get the following result.

Theorem 2. Let $A$ be a reversible $l-l$ method. Then $l_{A}=l$ if and only if $A$ has a unique left $l-l$ reciprocal.

Proof. If $A$ has a left $l-l$ reciprocal, it is unique if and only if the only bounded sequence which annihilates $A$ from the left is the zero sequence. (For, if one can choose a nonzero bounded sequence $t$ such that $t A=0$, then adding $t$ to any row yields a second left $l-l$ reciprocal. Conversely, if $A$ has two left $l-l$ reciprocals, their difference yields a nonzero bounded sequence which annihilates $A$ from the left.) If $l_{A}=l, 0$ is the only bounded left annihilator of $A[2$, Theorem 2, p. 359], and, by Theorem $1, A$ has a left $l-l$ reciprocal, which is therefore unique. On the other hand, if $A$ has a left $l-l$ reciprocal then, by Lemma $1, l$ is closed in $l_{A}$, and if this reciprocal is unique $l$ is dense in $l_{A}$ [2, Theorem 2, p. 359].

In a paper being prepared, the problem of $l_{A}=l$ will be further investigated.

4. Factorable methods. A matrix $A=\left(a_{n k}\right)$ is factorable if $a_{n k}$ $=a_{n} b_{k}$ for $k \leqq n$ and 0 for $k>n$. Let $E$ be an FK space containing the set of all finite sequences. An $l-l$ method $A$ will be called $E$-perfect if $\Delta$ is fundamental in $E \cap l_{A}$, where the closure is taken in the $l_{A^{-}}$ topology. For example, $A$ is 0 -perfect if and only if $A$ is $m_{r}$-perfect. The following result is proved in [3, Theorem 5].

Theorem. Let $A$ be a reversible $l-l$ method. If for every bounded sequence $t$ the condition $t A=0$ implies the condition

$$
\lim _{S} \sum_{k}\left|\sum_{n=1}^{S}\left(a_{n k}-a_{n, k+1}\right) t_{n}\right|=0,
$$

then $A$ is 0 -perfect. 
We now show that we may drop the assumption of reversibility for factorable $l-l$ methods.

For any sequence space $E$, let $E^{*}$ denote the set of sequences $t$ such that $\sum_{k} t_{k} x_{k}$ converges for every $x \in E$.

Theorem 3. Let $A$ be a factorable $l-l$ method with $a \in l$. If $b \in E^{*}$, then $A$ is E-perfect.

Proof. Let $f \in l_{A}^{\prime}$ such that $f=0$ on $l$. Then there exists a bounded sequence $t$ such that for all $x \in l_{A}$,

$$
f(x)=\sum_{n=1}^{\infty} t_{n} a_{n} \sum_{k=1}^{n} b_{k} x_{k}-\sum_{k=1}^{\infty} b_{k} x_{k} \sum_{n=k}^{\infty} t_{n} a_{n} .
$$

For each $h=1,2, \cdots$, let

$$
F_{h}(x)=\sum_{n=1}^{\infty} t_{n} a_{n} \sum_{k=1}^{n} b_{k} x_{k}-\sum_{k=1}^{h} b_{k} x_{k} \sum_{n=k}^{\infty} t_{n} a_{n} .
$$

Then $\lim _{h} F_{h}(x)=f(x)$ for each $x \in l_{A}$.

Now let $\epsilon>0$ be given and restrict $x$ to $E \cap l_{A}$. Then

$$
\left|F_{h}(x)\right|=\left|\sum_{n=h+1}^{\infty} t_{n} a_{n} \sum_{k=h+1}^{n} b_{k} x_{k}\right| \leqq \sum_{n=h+1}^{\infty}\left|t_{n} a_{n}\right| \cdot\left|\sum_{k=h+1}^{n} b_{k} x_{k}\right| .
$$

Let $M=\sum_{n=1}^{\infty}\left|t_{n} a_{n}\right|$. If $M=0,\left|F_{h}(x)\right|=0$ for each $h$. If $M \neq 0$, choose $H$ so that for $h \geqq H,\left|\sum_{k=n+1}^{n} b_{k} x_{k}\right|<\epsilon / M$ for all $n \geqq h+1$. Then for $h \geqq H,\left|F_{h}(x)\right|<\epsilon$. It follows that $f$ vanishes on $E \cap l_{A}$, so that $A$ is $E$-perfect.

That the converse of Theorem 3 is false follows by letting $E=s$, the FK space of all sequences, and letting $A$ be given by $a_{n}=2^{-n}$ and $b_{k}=2^{k} . l_{A}=l$ since the two-sided reciprocal for $A$ is $l-l$ and yet $b$ is not in $s^{*}$.

\section{BIBLIOGRAPHY}

1. S. Banach, Theorie des opérations linéaries, Monografje Matematyczne, Warsaw, 1932.

2. H. I. Brown and V. F. Cowling, On consistency of $l-l$ methods of summation, Michigan Math. J. 12 (1965), 357-362.

3. E. Jürimäe, Certain questions of inclusion and compatibility of absolute summability methods, Tartu Riikl. Utl. Toimetised 150 (1964), 132-143. (Russian)

4. K. Knopp and G. G. Lorentz, Beiträge zur absoluten Limitierung, Arch. Math. 2 (1949), 10-16.

5. A. Wilansky, Functional analysis, Blaisdell, New York, 1964.

State University of New York at Albany 